\title{
Status der Kosmetik-Reglementierung in Südkorea
}

\begin{abstract}
Südkorea gehört zu den zehn größten Kosmetikmärkten der Welt und gilt als globales Zentrum für Innovationen in der Kosmetik. "K-Beauty“ ist auf dem Vormarsch, südkoreanische Produkte beeindrucken durch ihre Wirksamkeit, Verpackung und sensorische Attraktivität und inspirieren damit auch zunehmend westliche Marken.

Um einen Beitrag zum Verbraucherschutz zu leisten, der die Entwicklung der Kosmetikindustrie nicht behindert, erließ die südkoreanische Regierung im Jahre 2000 eine übergreifende Reglementierung für Kosmetika, die als „Cosmetics Act" bekannt ist. Das mehrfach überarbeitete Gesetz sieht Maßnahmen für die Herstellung, den Import und den Verkauf von Kosmetika und kosmetischen Inhaltsstoffen vor, einschließlich detaillierter Anforderungen an die Kennzeichnung und Reklame der Produkte. In einer Revision vom April 2020 wurden noch einmal wesentliche Änderungen im Bereich der Sicherheitsstandards und Produktkennzeichnung veranlasst, die Marktteilnehmer entlasten.
\end{abstract}

\section{Die Kosmetik-Gesetzgebung vor April 2020}

Der südkoreanische Kosmetikmarkt repräsentiert etwa 2,8 $\%^{1}$ des globalen Marktes und hat heute einen Wert von etwa 10 Milliarden US\$ mit einer geschätzten jährlichen Wachstumsrate (CAGR) von 4,95 \% im Zeitraum $2017-2030^{2}$.

Vor der Einführung des "Cosmetics Act” ${ }^{3}$ (Gesetz Nr. 17250) im Jahre 2000 wurden Vorschriften zu Kosmeti$\mathrm{ka}^{4}$ in der Gesetzgebung für „Pharmazeutische Angelegenheiten" erlassen. Seitdem werden die Vorschriften für kosmetische Produkte und Rohstoffe vom Ministerium für Lebensmittel- und Arzneimittelsicherheit ${ }^{5}$ („MFDS“) verabschiedet und verwaltet. Das neue Gesetz wurde in Kraft gesetzt, um die Wettbewerbsfähigkeit der heimischen Kosmetikindustrie zu stärken und mit dem internationalen Trend der Regulierungen Schritt zu halten. Das Kosmetikgesetz hat seit dem Jahre 2000 verschiedene Änderungen durchlaufen, wie z. B. die Unterteilung verwandter Vorschriften, die Annahme von GMP-Standards für Kosmetika (cGMP $\left.{ }^{6}\right)$ und die Aufnahme von Zertifizierungsstandards für Naturund Biokosmetika.

Der Gesetzestext umfasst hauptsächlich die rechtlichen Verpflichtungen speziell für Importeure von Kosmetika, um nicht-koreanischen Herstellern zu helfen, ihre Produkte nach Südkorea zu exportieren. Die Verpflichtungen hängen

DOI: $10.21552 /$ stoffr/2020/4/3

* Doris Peters ist Geschäftsführerin der Consortia Management GmbH in Worms, E-Mail: peters@consortia-management.com, Jae-Seong Choi ist Zweigstellenleiter der Chemservice Asia Co. Ltd., E-Mail: js.choi@chemservice-asia.com.

1 International Trade Administration, South Korea commercial guide, abrufbar im Internet unter www.trade.gov/knowledge-product/korea- cosmetics\#: :text=South $\% 20$ Korea $\% 20$ is $\% 20$ the $\% 209$, respectively\%20from\%20the\%20previous\%20year (letzter Zugriff 24.10.2020).

2 Goldstein Market Intelligence, South Korea Cosmetics Market Report By Product Type (Fragrances, Skin Care, Make-Up, Hair Care, Hygiene, Oral Cosmetics), By Pricing (Low and Medium Priced \& Premium-Priced Cosmetics), By Gender (Male \& Female), By Distribution Channel With COVID-19 impact \& Forecast 2017-2030, 2020, abrufbar im In- von der Art und der Kategorie des kosmetischen Produkts $a b$.

\section{Kategorien von kosmetischen Produkten}

Nach dem Kosmetikgesetz werden kosmetische Produkte in „allgemeine Kosmetika“ und „funktionelle Kosmetika“ unterteilt. Im Gegensatz zu den allgemeinen Kosmetika, bei denen Hersteller oder Importeure ihre Produkte ohne Registrierung vermarkten dürfen, aber einer Überwachung nach dem Inverkehrbringen unterliegen, ist es für Produkte, die der Gruppe der funktionellen Kosmetika angehören, obligatorisch, vor der Markteinführung ein Registrierungsverfahren bezüglich ihrer Sicherheit, Wirksamkeit und Funktion durch das MFDS zu durchlaufen. Je nach Verwendungszweck, wie z. B. Säuglinge, Bäder, Duftstoffe, Waschen und Färben, werden funktionelle Kosmetika weiter in Kategorien unterteilt (Tabelle 1) und unterliegen dementsprechend unterschiedlichen Anforderungen.

Funktionelle Kosmetika sind in Korea nicht zu verwechseln mit Verbrauchsgütern, die als Quasi-Arzneimittel eingestuft werden (wie z. B. Damenbinden, Handdesinfektionsmittel, Antiseptika, Zahnpasta, Mundwasser, usw.) und nach dem Arzneimittelgesetz kontrolliert werden. Sie unterliegen unterschiedlichen Registrierungspflichten. Bevor sie

ternet unter www.goldsteinresearch.com/report/korean-cosmetics-market-report-south-korea-industry-analysis (letzter Zugriff 24.10.2020).

3 Cosmetics Act (Act No. 17250), Überarbeitung des MFDS in Kraft seit 7.4.2020.

4 Kosmetika werden in Südkorea definiert als Produkte, die auf den menschlichen Körper aufgetragen oder aufgesprüht werden, um Haut und Haar zu reinigen, zu verschönern, zu verändern, aufzuhellen, zu pflegen oder die Gesundheit von Haut und Haar zu fördern.

5 Ministry of Food and Drug Safety (MFDS), Republic of Korea, abrufbar im Internet unter www.mfds.go.kr/eng/index.do (letzter Zugriff 24.10.2020).

$6 \quad \mathrm{cGMP}=$ Gute Herstellungspraxis für Kosmetika (Cosmetics Good Manufacturing Practice). 
Tabelle 1: Kategorien von funktionellen Kosmetika ${ }^{a}$

\begin{tabular}{|c|c|c|}
\hline Anwendungsbereich & Kategorie & Bemerkungen \\
\hline \multirow[t]{7}{*}{ Haut } & Hautaufhellung & $\begin{array}{l}\text { durch Verhinderung oder Verblassen der Melaninpigmentie- } \\
\text { rung }\end{array}$ \\
\hline & Anti-Falten & \\
\hline & Bräunung & \\
\hline & UV-Schutz & \\
\hline & Linderung von Akne & nur „rinse-off“ \\
\hline & Linderung atopischer Haut & \\
\hline & Behandlung von Dehnungsstreifen & \\
\hline \multirow[t]{3}{*}{ Haar } & Färben (inkl. Bleichen) & exkl. temporäre Haarfärbemittel \\
\hline & Haarentfernung & exkl. physikalische Haarentfernung \\
\hline & Linderung von Alopezie (Haarausfall) & exkl. physikalische Haarverdickung (Beschichtung) \\
\hline
\end{tabular}

a Enforcement Rule of the Cosmetics Act (Ordinance of the Prime Minister No. 1636), Art. 2 (Scope of Functional Cosmetics).

Güter nach Korea einführen, sollten Importeure sich immer über die neuesten Vorschriften und Kategorisierungen von Konsumgütern und funktionellen Kosmetika informieren, um dem derzeit geltenden Recht zu entsprechen und unnötige Maßnahmen oder gar Strafen zu vermeiden.

\section{Rechtliche Anforderungen beim Import von Kosmetika}

Insgesamt wurden die gesetzlichen Verpflichtungen zur Herstellung, zum Import, zum Vertrieb oder zum Verkauf von kosmetischen Mitteln seit der Einführung des Kosmetikgesetzes vereinfacht, um die Wettbewerbs- und Marktfähigkeit zu sichern. Die Behörden haben jedoch die Sicherheitsprüfungen von Produkten und Rohstoffen aus Gründen des Verbraucherschutzes verstärkt. Die Zuständigkeiten für das Qualitätsmanagement und die Kommunikation bezüglich der Sicherheit von Produkten müssen von jedem autorisierten Akteur in der Lieferkette strikt eingehalten werden. Importeure sollten bspw. eine Selbstkontrolle der Produktqualität von importierten Kosmetika durchführen und dürfen keine Produkte auf den koreanischen Markt bringen, die verbotene Inhaltsstoffe enthalten. Im Falle eines Verstoßes kann das MFDS die Produkte durch eine Rückrufaktion mit sofortiger Wirkung vom Markt nehmen, was sich in Korea sehr schadhaft auf das zukünftige Geschäft und den Ruf nicht nur des Importeurs, sondern auch des Herstellers auswirkt.

\section{a. Import-Verfahren}

Die nachstehende Abbildung 1 zeigt den Ablauf des Imports funktioneller Kosmetika nach dem Kosmetikgesetz, von der Gewerbeanmeldung über die Kennzeichnung bis hin zum Vertrieb und der Vermarktung.

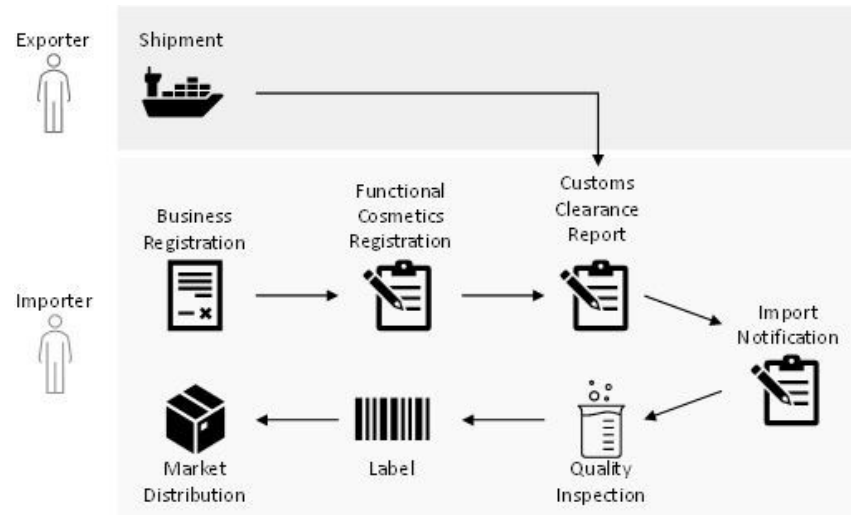

Abbildung 1: Import-Prozess

\section{aa. Registrierung von Unternehmen}

Jede Person oder Firma, die beabsichtigt, Kosmetika in Korea herzustellen, zu importieren oder zu verkaufen, muss zunächst ein entsprechendes Gewerbe anmelden. Dabei ist wie folgt zu unterscheiden:

- Produktionsgewerbe;

- verantwortungsvolles Vertriebsgewerbe für Kosmetika;

- Verkauf von "Customized Cosmetics“. 
Beim Import von Kosmetika ist der Importeur angehalten, sich als „verantwortlicher Kosmetikdistributor" (sogenannter Zulassungsinhaber, MAH) zu registrieren, der für die rechtlich einwandfreie Einhaltung der Einfuhrzölle für Kosmetika nach dem Kosmetikgesetz verantwortlich ist. Daher ist es für den nicht-koreanischen Hersteller wichtig zu überprüfen, ob der Importeur entsprechend der Gewerbeanmeldung ordnungsgemäß autorisiert ist. Weiterhin muss der Hersteller dem Importeur die erforderlichen Unterlagen zur Verfügung stellen, um den Verpflichtungen beim Import von Kosmetikprodukten nach koreanischem Recht nachzukommen.

\section{bb. Registrierung von funktionellen Kosmetika}

Wie unter I.1 beschrieben, unterliegen funktionelle Kosmetika einer Registrierung durch das MFDS, um vor der Herstellung oder Einfuhr nach Korea eine Genehmigung zu erhalten. Die Dossiers werden bewertet, um die Sicherheit und Wirksamkeit nachzuweisen und das Qualitätsmanagement gemäß den im $\mathrm{KFCC}^{7}$ festgelegten Prüfstandards zu verifizieren (Tabelle 2). Die Registrierungsdossiers müssen der Behörde in koreanischer Sprache vorgelegt werden.

Einige der Dossieranforderungen (wie z. B. Herkunft und Informationen zum Entwicklungshintergrund sowie Daten aus klinischen Studien und Wirksamkeitsstudien) können unter bestimmten Bedingungen gemäß Anhang 4 des „Standard of Functional Cosmetics Evaluation (MFDS Public Notification No. 2019-47)" ausgenommen werden, wenn ein funktionelles Produkt nur Inhaltsstoffe enthält, die bereits in diesem Standard aufgeführt sind.

\section{cc. Zollabfertigung}

Nach der Bewilligung der Registrierung der funktionellen Kosmetika ist nach der physischen Einfuhr des Produkts nach Korea die Zollabfertigung erforderlich. Dazu ist eine Importmeldung bei der KPTA ${ }^{8}$ einzureichen, um ein Zollabfertigungsverfahren mit den folgenden Anforderungen einzuleiten:

- Bericht der Zollabfertigung (vom Importeur im EDI ${ }^{9}$-Format einzureichen);

- Zertifikat der Herstellung;

- Dokumente zu TSE ${ }^{10}$ (und/oder BSE ${ }^{11}$ ).

$7 \quad$ KFCC $=$ Korean Functional Cosmetics Codex (MFDS Public Notice No. 2018-111).

8 Korea Pharmaceutical Traders Association, abrufbar im Internet unter www.kpta.or.kr/eng/main/main.asp (letzter Zugriff 24.10.2020).

$9 \mathrm{EDI}=$ Elektronischer Datenaustausch (Electronic Data Interchange Method).

10 TSE = Übertragbare spongiforme Enzephalopathie (Transmissible Spongiform Encephalopathy).

11 BSE $=$ Bovine spongiforme Enzephalopathie (Bovine Spongiform Encephalopathy).

$12 \mathrm{INCI}=$ Internationale Nomenklatur für kosmetische Inhaltsstoffe (International Nomenclature Cosmetic Ingredient).
Für die Einfuhr funktioneller Kosmetika ist zusätzlich ein Zulassungsschreiben der Produktregistrierung vom MFDS erforderlich. Nach der Zollabfertigung werden die Produkte i. d. R. in einem Lagerhaus aufbewahrt, bis die Qualitätsprüfung abgeschlossen ist und das Genehmigungsschreiben ausgestellt wird.

Der Gesamtprozess der Zollabfertigung von Rohstoffen ist vergleichbar mit dem Prozess für Fertigprodukte. Wenn ein einzelner Rohstoff importiert wird, ersetzt der INCI ${ }^{12}$ Name den Produktnamen in der Dokumentation. Im Falle von Mischungen verschiedener Rohstoffe reicht es aus, einen Bericht für die Mischung einzureichen. Der INCI-Name jedes Inhaltsstoffes ist anzugeben, und anstelle der Rohstoff-Codierung gilt der Code des Harmonisierten Systems (HS-Code) seines abgeleiteten kosmetischen Fertigerzeugnisses.

Rohstoffe, einschließlich deren Mischungen, erfordern vom Importeur in Korea keine Gewerbeanmeldung gemäß dem Kosmetikgesetz. Jedes Unternehmen kann Rohstoffe importieren und an registrierte Hersteller weiterverkaufen, nachdem eine Selbstinspektion der Rohstoffe durchgeführt wurde.

\section{dd. Überprüfung der Produktqualität}

Die Prüfmethoden oder die Häufigkeit der Qualitätskontrolle eines Produkts sind nach dem Kosmetikgesetz nicht vorgeschrieben, aber der Importeur ist als MAH verpflichtet, eine Selbstkontrolle des Produkts durchführen, um sicherzustellen, dass die eingeführten Produkte die Sicherheitsstandards des Kosmetikgesetzes erfüllen. Dabei sind eine Negativliste der Inhaltsstoffe und die Grenzwerte der Prüfparameter zu beachten. Bei funktionellen Kosmetika sind nach den KFCC-Standards pro Kategorie unterschiedliche Parameter zu verifizieren. Falls der Hersteller cGMP-zertifiziert ist oder die koreanischen cGMP-Bedingungen durch eine Vor-Ort-Inspektion der koreanischen Behörden erfüllt werden können, kann auf die Verpflichtung des Importeurs zur Qualitätskontrolle verzichtet werden. Wenn der Importeur die geforderten Prüfungen selbst nicht durchführen kann, kann er mit dieser Aufgabe auch qualifizierte Labors oder Institute beauftragen.

\section{ee. Etikettierung}

Der finale Schritt, der vor dem Verkauf der Kosmetikprodukte auf dem koreanischen Markt zu beachten ist, ist die ordnungsgemäße Kennzeichnung der Produkte. Selbstverständlich sind Etiketten bereits auf den importierten Kosmetika in der Sprache angebracht, die je nach Standort des Herstellers den einschlägigen Vorschriften entspricht. Zusätzlich müssen jedoch Etiketten in koreanischer Sprache angebracht werden, damit koreanische Verbraucher die Informationen auf den Etiketten in ihrer Landessprache leicht überprüfen können. Der koreanische Importeur muss seine Koordinaten (Name, Adresse usw.) angeben, und darüber hinaus sollte auf dem Etikett die Nummer der Herstellungs- 
Tabelle 2: Anforderungen an die Dossiers für die Registrierung funktioneller Kosmetika

\begin{tabular}{|l|l|l|}
\hline Kategorien & Informationsanforderung & Bemerkungen \\
\hline Klinische \& Wirksamkeitsinformationen & Herkunft \& Entwicklungshintergrund & \\
\cline { 2 - 3 } & $\begin{array}{l}\text { Klinische \& wirksamkeitsbezogene Studien- } \\
\text { daten }\end{array}$ & $\begin{array}{l}\text { kann ausgenommen werden, wenn Inhalts- } \\
\text { stoffe gelistet sind }\end{array}$ \\
\cline { 2 - 3 } & Nachweise zu SPFb, PAc-Bewertung & Nur für SPF-Produkte \\
\hline Testmethode und Standard & $\begin{array}{l}\text { Prüfgegenstand, Validierung der Prüfmetho- } \\
\text { de }\end{array}$ & \\
\hline
\end{tabular}

a Standards for Evaluation Dossiers of Functional Cosmetics (MFDS Public Notice No. 2019-47), Annex 4: Types of functional cosmetics and maximum limit of ingredients for data submission exemption.

b $\quad$ SPF $=$ Sonnenschutzfaktor (Sun Protection Factor).

c $\quad P A=$ UVA-Schutzwirkung (Protection Factor of UVA).

charge in Verbindung mit dem Ergebnis der Qualitätsprüfung des Produkts nach dem Import angegeben werden.

Tabelle 3: Etikettierung gemäß Inhalt (Gewicht/Volumen)

\begin{tabular}{|c|c|}
\hline $\begin{array}{l}\text { Gewicht (oder Volu- } \\
\text { men) }\end{array}$ & Geforderte Etikettierung \\
\hline $\begin{array}{l}10 \mathrm{~g} \text { (oder } \mathrm{ml}) \text { oder we- } \\
\text { niger }\end{array}$ & $\begin{array}{l}\text { Produktname } \\
\text { Firmenname des Importeurs } \\
\text { Chargen-Nummer } \\
\text { Mindesthaltbarkeitsdatum } \\
\text { Verkaufspreis }^{\mathrm{a}}\end{array}$ \\
\hline $11-50 \mathrm{~g}$ (oder ml) & $\begin{array}{l}\text { Zusätzlich zu den oben genannten An- } \\
\text { forderungen: } \\
\text { Adresse des Importeurs \& Kontaktinfor- } \\
\text { mation } \\
\text { Ursprungsland } \\
\text { Firmenname des Herstellers \& Adresse } \\
\text { Nettogewicht/-volumen } \\
\text { biste der Inhaltsstoffe mit max. Grenz- } \\
\text { werten }^{b} \\
\text { Sicherheitshinweise }^{b} \\
\text { Barcode }^{b} \\
\text { Separate Entladungsmarkierung }^{c}\end{array}$ \\
\hline mehr als $50 \mathrm{~g}$ (oder ml) & $\begin{array}{l}\text { Zusätzlich zu den oben genannten Anfor- } \\
\text { derungen: } \\
\text { Vollständige Liste der Inhaltsstoffe }{ }^{b} \text { und } \\
\text { Entladungsmarkierung }\end{array}$ \\
\hline
\end{tabular}

a Vom Endverkäufer gekennzeichnet. Falls eine Sekundärverpackung vorhanden ist, kann der Verkaufspreis auf der Primärverpackung entfallen.

b Wenn eine Sekundärverpackung vorhanden ist, können diese Parameter auf der Primärverpackung weggelassen werden.

c Eine separate Entladungsmarkierung kann weggelassen werden, wenn das Gewicht (oder Volumen) des Inhalts weniger als $30 \mathrm{~g}$ (oder $\mathrm{ml}$ ) beträgt.

\section{Kosmetik-GMP (cGMP)}

In Korea wurde cGMP ursprünglich im Jahre 1990 im Rahmen des „Pharmaceutical Affairs Act" eingeführt, wegen des begrenzten Nutzens für die Industrie aber nicht aktiv um- gesetzt. Für Produktionsstätten, die ihre Anlagen gut und in Übereinstimmung mit den internationalen GMP-Standards führten, war die Pflicht zur zusätzlichen Inspektion der Qualität in Korea wenig sinnvoll und behinderte überdies die internationale Wettbewerbsfähigkeit der einheimischen Kosmetikhersteller. Nach Jahren stetiger Forderungen der Industrie nach Änderungen der cGMP-Verpflichtungen führte das MFDS 2009 eine Überarbeitung der bestehenden cGMP-Verordnung ein, nicht nur um die Qualität der Kosmetikherstellung besser steuern zu können, sondern auch, um die internationale Wettbewerbsfähigkeit der einheimischen Unternehmen zu verbessern. Heute sind die Standardkriterien der cGMP vergleichbar mit ISO $22716^{13}$, dem internationalen Standard mit umfassenden Kriterien für die Herstellung von Kosmetika.

Importeure können von der Inspektion der Produktqualität befreit werden, wenn Behörden im Rahmen einer Standortinspektion die Anlagen des ausländischen Herstellers inspizieren und dabei die Kriterien des koreanischen cGMPStandards zugrunde legen. Da der überarbeitete cGMP-Standard viel mit internationalen Standards wie der cGMP-Norm der EU oder ISO 22716 gemein hat, erfüllen cGMP-zertifizierte EU-Hersteller die koreanischen Standards ebenso und haben dadurch einen leichteren Marktzugang und eine bessere Wettbewerbsfähigkeit auf dem koreanischen Markt.

\section{Zertifizierung von Natur- und Biokosmetika}

Abgesehen von der Kategorisierung nach der Funktion oder dem Verwendungszweck können sowohl allgemeine als

13 International Organization for Standardization, ISO 22716:2007 Cosmetics - Good Manufacturing Practices (GMP) - Guidelines on Good Manufacturing Practices, abrufbar im Internet unter www.iso.org/standard/36437.html (letzter Zugriff 18.11.2020). 
auch funktionelle Kosmetika in Abhängigkeit von den Inhaltsstoffen als Naturkosmetika und Biokosmetika bezeichnet werden. Nach dem Kosmetikgesetz ist eine Naturkosmetik definiert als ein kosmetisches Produkt, das $95 \%$ oder mehr natürliche oder aus natürlichen Quellen stammende Inhaltsstoffe enthält. Biokosmetika sind kosmetische Produkte, die $10 \%$ oder mehr organische Inhaltsstoffe enthalten.

Mit dem Interesse der Verbraucher an den in Kosmetika verwendeten Inhaltsstoffen stieg auch die Nachfrage nach Kosmetika mit organischen oder natürlichen Inhaltsstoffen. Unangemessene Reklame und die Verwendung nicht überprüfter Inhaltsstoffe riefen Bedenken hinsichtlich der Sicherheit der Verbraucher hervor und erforderten die Einführung geeigneter Standards und eines Zertifizierungssystems für Bio- und Naturkosmetika. Das Zertifizierungssystem für Biokosmetika in Korea wurde erstmals 2015 unter dem Namen „Regulations on the Standards of Organic Cosmetics (MFDS Public Notice No. 2014-200)“ mit einer Positivliste von Bio-Inhaltsstoffen und den Standards für Herstellungsprozesse und -anlagen in Kraft gesetzt. Die Verordnung entsprach dem Sicherheitsbedürfnis der Verbraucher bei Biokosmetika, indem sie die entsprechende Kennzeichnung nur für Produkte zuließ, die vom MFDS zertifiziert wurden. Seit Juli 2019 gilt die Überarbeitung der Verordnung als „Verordnung über die Standards für Naturkosmetika und Biokosmetika (MFDS-Veröffentlichung Nr. 2019-66)“ und enthält nun zusätzlich die Definition von Naturkosmetika neben Biokosmetika sowie eine Aktualisierung der Standards für Inhaltsstoffe und Kategorien zur Zulassung. Da zum 1.1.2021 die Übergangsfrist ${ }^{14}$ der Kennzeichnung für Biokosmetika nach der vorherigen Verordnung für die Kennzeichnung der Produkte abläuft, müssen Lieferanten auf die Aktualisierungen der Kennzeichnung achten.

\section{II. Änderungen in der Kosmetik-Gesetzgebung seit April 2020}

Mit der Revision vom April 2020 wurden wesentliche Änderungen der Kosmetik-Regulierung zur Entlastung der Marktteilnehmer umgesetzt. Dies hat nicht nur die Aufmerksamkeit inländischer Unternehmen geweckt, sondern auch die nicht-koreanischer Hersteller, die ihre Produkte exportieren wollen. Die revidierte Gesetzgebung zielt darauf ab, die Sicherheit der Konsumenten weiter zu verbessern. Wesentliche Änderungen betreffen das Geschäft mit „Customized Cosmetics" sowie die Sicherheitsstandards

14 Regulations on the Standards of Natural Cosmetics and Organic Cosmetics (MFDS Public Notice No. 2019-66) - Supplementary provision Article 2 (Transitional measures related to labeling of organic cosmetics).

15 Regulations on Cosmetics Safety Standards (MFDS Public Notice No. 2020-12) und die neuen Verpflichtungen zur Kennzeichnung der Produkte.

\section{Einführung des "Customized Cosmetics"- Systems}

Eine „customized“ oder maßgeschneiderte Kosmetik ist ein Kosmetikprodukt, das von einem zertifizierten „technischen Manager für maßgeschneiderte Kosmetik" in einem Einzelhandelsgeschäft auf der Grundlage persönlicher Vorlieben oder des Hauttyps angepasst werden kann.

Die Einführung des „Customized Cosmetics“-Systems im koreanischen Markt im Jahr 2020 ist von größtem Interesse. Zuvor verbot das Kosmetikgesetz das Mischen und/oder Abfüllen von Kosmetika in kleinen Mengen in Einzelhandelsgeschäften, während die Nachfrage nach verschiedenen Optionen für kundenspezifische Produkte und personalisierte Dienste weiter zunahm. Daher war eine neue Gesetzgebung erforderlich, um die Nachfrage der Verbraucher zu bedienen und gleichzeitig ihre Sicherheit zu gewährleisten. Nach dem revidierten Kosmetikgesetz darf heute ein zertifizierter „Technischer Manager für maßgeschneiderte Kosmetik" Bestandteile von Kosmetikprodukten legal abfüllen und/oder mischen. Eine Gewerbeanmeldung für "Customized Cosmetics" ist obligatorisch, um ein Geschäft mit zertifiziertem Personal betreiben zu dürfen.

Das „Customized Cosmetics“-System stellt eine große Veränderung und Erweiterung des koreanischen Kosmetikmarktes dar, da nicht nur Unternehmen, die direkt mit Kosmetikprodukten zu tun haben, ein Interesse an diesem Geschäft haben, sondern auch Hautpflegeexperten und Schönheitssalons. Darüber hinaus wird erwartet, dass auch $3 \mathrm{D}$ Technologien, KI und Biotechnologien mit diesem Geschäftsbereich kombiniert werden können, um den Markt weiter zu entwickeln.

\section{Aktualisierter Sicherheitsstandard}

Mit der letzten Revision des Kosmetikgesetzes wurden auch die Sicherheitsstandards ${ }^{15}$ in Form einer Negativliste von Inhaltsstoffen, deren Verwendung verboten oder eingeschränkt sind, überarbeitet. So werden bspw. Tagetes erecta-, Tagetes minuta- und Tagetes patula-Blütenextrakte und -öle, die als Duftinhaltsstoffe vieler Parfüms weit verbreitet waren, unter der koreanischen Verordnung neu kontrolliert. Insbesondere Tagetes erecta-Blütenextrakt und -öl, das bereits auf dem EU-Kosmetikmarkt verboten ist, wird in die Negativliste aufgenommen. Darüber hinaus sind Tagetes minuta-Extrakt und -Öl sowie Tagetes patula-Extrakt und -Öl mit der gleichen Konzentrationsgrenze wie in der EU (0,1 $\%$ in „rinse-off“-Produkten und o,01 \% in „Leave-on“-Produkten) als beschränkt verwendbar aufgeführt, d. h. diese Inhaltsstoffe werden durch internationale Standards kon- 
trolliert. Das MFDS hat diese Änderungen in die Überarbeitung des Gesetzes aufgenommen. Darüber hinaus wurde in Art. 3 der Verordnung eine Positivliste der Inhaltsstoffe für „Customized Cosmetics“ neu aufgenommen.

Schönheitsseifen in fester Form wurden von der vorherigen Kategorisierung als Industrieprodukt, das unter dem „Electronical Appliances and Consumer Products Safety Control Act" (Act No. 15338) reguliert war, in das Kosmetikgesetz übernommen, in Einklang mit anderen Arten von Seifen (z. B. Flüssigseife, Körperreinigungsmittel usw.), die bereits unter dem Kosmetikgesetz kontrolliert werden. Aufgrund dieser Änderung für Schönheitsseifen wurden neue Prüfstandards und -methoden im Anhang $4^{16}$ der Verordnung festgelegt. Darüber hinaus sind verbotene Stoffe ${ }^{17}$, die nach dem K-REACH-Act ${ }^{18}$ für Chemikalien neu beurteilt wurden, heute laut Kosmetikgesetz auch verboten.

\section{3. Änderungen der Anforderungen zur Etikettierung}

Unter den im Jahr 2020 überarbeiteten Vorschriften können die Änderungen der Anforderungen zur Etikettierung als die relevantesten für nicht-koreanische Hersteller und Vertreiber angesehen werden. Der „Standard on Marking Cosmetics Usage Precautions and Fragrance Allergens“ (Standard zur Kennzeichnung von Vorsichtsmaßnahmen bei der Verwendung von Kosmetika und Duftstoffallergenen) verlangt, dass alle Kosmetika auf ihren Etiketten das Vorhandensein der 25 bekanntesten allergieauslösenden chemischen Substanzen mit ihrem INCI-Namen in koreanischer Sprache anzeigen müssen, falls die Konzentrationen in den Endprodukten die vorgegebenen Konzentrationsschwellenwerte überschreiten (Tabelle 4). Es wird erwartet, dass Beschwerden von Verbrauchern, die auf bestimmte Inhaltsstoffe allergisch reagieren, durch diese Maßnahme stark reduziert werden können. Allergene wurden in der Vergangenheit nur als „Duftstoffe“ gekennzeichnet.

\section{Tabelle 4: Duftstoffallergene}

\begin{tabular}{|l|l|l|}
\hline Nr. & Chemischer Name & CAS Nr. \\
\hline 1 & Amyl Cinnamal & $122-40-7$ \\
\hline 2 & Benzyl Alkohol & $100-51-6$ \\
\hline 3 & Cinnamyl Alkohol & $104-54-1$ \\
\hline 4 & Citral & $5392-40-5$ \\
\hline 5 & Eugenol & $97-53^{-0}$ \\
\hline 6 & Hydroxycitronellal & $107-75^{-5}$ \\
\hline 7 & Isoeugenol & $97-54-1$ \\
\hline
\end{tabular}

\begin{tabular}{|c|c|c|}
\hline Nr. & Chemischer Name & CAS Nr. \\
\hline 8 & Amyl Cinnamal Alkohol & $101-85-9$ \\
\hline 9 & Benzyl Salicylate & $118-58-1$ \\
\hline 10 & Cinnamal & $104-55^{-2}$ \\
\hline 11 & Coumarin & $91-64-5$ \\
\hline 12 & Geraniol & $106-24-1$ \\
\hline 13 & Anise Alkohol & $105^{-1} 3^{-5}$ \\
\hline 14 & Benzyl Cinnamate & $103-41-3$ \\
\hline 15 & Farnesol & $4602-84-0$ \\
\hline 16 & Butylphenyl Methypropional & $80-54-6$ \\
\hline 17 & Linalool & $78-70-6$ \\
\hline 18 & Benzyl Benzoate & $120-51-4$ \\
\hline 19 & Citronellol & $106-22-9$ \\
\hline 20 & Hexyl Cinnamal & $101-86-0$ \\
\hline 21 & Limonene & $5989-27-5$ \\
\hline 22 & Methyl 2-Octynoate & $111-12-6$ \\
\hline 23 & Alpha-Isomethyllonone & $127^{-51-5}$ \\
\hline 24 & Evernia Prunastri (Oakmoss) Extrakt & 90028-68-5 \\
\hline 25 & Evernia Furfuracea (Treemoss) Extrakt & $90028-67-4$ \\
\hline
\end{tabular}

\section{Fazit}

Seit der Einführung des „Cosmetics Act“ in Südkorea im Jahre 2000 wurde das Gesetz mehrfach überarbeitet, zuletzt im April 2020. Das MFDS hat damit Anstrengungen unternommen, die Wettbewerbsfähigkeit der einheimischen Kosmetikindustrie zu verbessern, mit den internationalen Regulierungen Schritt zu halten und gleichzeitig den Verbraucherschutz zu gewährleisten. Im Rahmen der Kosmetik-Gesetzgebung in Korea gibt es so ein definiertes Verfahren für den Import funktioneller Kosmetika, von der Gewerbeanmeldung des Importeurs über die Registrierung des Produkts selbst bis hin zur Kennzeichnung und Vermarktung.

16 Test method for safety management of consumer cosmetics.

17 Designation of Restricted, Prohibited Chemical Substances (Ministry of Environment Public Notice No. 2019-214).

18 Act on Registration, Evaluation, etc. of Chemicals (Act No. 17326, revision enforced on $5^{\text {th }}$ May 2020 by Ministry of Environment). 
Die Gesetzgebung verpflichtet Hersteller und Importeure auch zur Erfüllung von cGMP-Standards.

Für Kosmetikprodukte, die als Natur- oder Biokosmetika vermarktet werden, gibt es ein Zertifizierungssystem, um falsche Etikettierung oder Reklame zu verhindern. Die Gesetzgebung hat außerdem Risikoeinstufungen und Klassifizierungsstandards für potenziell gefährliche Kosmetikprodukte oder Inhaltsstoffe eingeführt, die in Zukunft möglicherweise vom Markt genommen werden müssen. Besonderes Augenmerk wurde auf Säuglings- und Kinderkosmetika gelegt, indem detaillierte Altersstandards und festgelegte Kriterien für die Werbung hinzugefügt wurden, um jegliche Sicherheitsprobleme zu vermeiden. Darüber hinaus sorgen regelmäßige Umfragen unter Verbrauchern sowie die Festlegung von Verfahren zur Reduzierung von Risikofaktoren.

Jüngste bedeutende Änderungen des Kosmetikgesetzes betreffen die Einführung des Systems zur Herstellung von kundenspezifischen Kosmetika, das es zertifiziertem Personal erlaubt, Bestandteile von Kosmetikprodukten abzufüllen oder zu mischen. Sie betreffen eine Aktualisierung der Sicherheitsstandards mit einer Negativliste von Bestandteilen, deren Verwendung verboten oder eingeschränkt sind, einer Positivliste von Bestandteilen für kundenspezifische Kosmetika sowie die Kennzeichnung von 25 bekannten Allergenen auf den Etiketten der Produkte mit ihrem INCI-Namen in koreanischer Sprache.

Mit den fortlaufenden Überarbeitungen der Regulierung kann festgehalten werden, dass das MFDS sich zum Ziel gesetzt hat, in Korea einen Markt zu schaffen, in dem Bedenken der Verbraucher bezüglich der sicheren Verwendung von hochwertigen Kosmetika minimiert oder sogar ausgeräumt werden können und gleichzeitig das Marktwachstum unterstützt wird. Aufgrund der zahlreichen Überarbeitungen sollten sich Importeure allerdings immer mit den aktuell geltenden Vorschriften vertraut machen. 